\title{
Práticas discursivas e produção de sentidos: análise da aplicação em Saúde Mental no Brasil
}

\author{
Discursive practices and production of meanings: analysis of the \\ application in Mental Health in Brazil
}

\section{Prácticas discursivas y producción de sentidos: análisis de la aplicación en Salud Mental en Brasil}

\author{
Amanda Pereira Risso Saad ${ }^{1, a}$ \\ amanda.saad@hotmail.com | http://orcid.org/oooo-0001-8527-4.366
}

Paulo Roberto Haidamus de Oliveira Bastos ${ }^{1, b}$

phaidamus43@gmail.com | http://orcid.org/o000-0002-8885-1461

Geisa Alessandra Cavalcante de Souza ${ }^{2, c}$

geisaacs@hotmail.com | http://orcid.org/o000-0002-2019-3239

\footnotetext{
${ }^{1}$ Universidade Federal de Mato Grosso do Sul, Programa de Pós-Graduação Stricto Sensu em Saúde e Desenvolvimento na Região Centro-Oeste. Campo Grande, MS, Brasil.

2 Universidade Federal de Mato Grosso do Sul, Programa de Pós-Graduação Stricto Sensu em Saúde da Família.

Campo Grande, MS, Brasil.

a Mestrado em Saúde e Desenvolvimento na Região Centro-Oeste pela Universidade Federal de Mato Grosso do Sul.

Doutorado em Educação pela Pontifícia Universidade Católica de São Paulo.

'Mestrado em Saúde da Família pela Universidade Federal de Mato Grosso do Sul.
}

\section{Resumo}

Este estudo analisou a aplicação das práticas discursivas e produção de sentidos na descrição e interpretação dos sentidos dados a diferentes fenômenos em saúde mental no Brasil. Trata-se de uma revisão bibliográfica baseada na literatura especializada, a partir da consulta a estudos científicos selecionados utilizando a Biblioteca Virtual em Saúde (BVS) com as palavras-chave 'práticas discursivas' e 'produção de sentidos', no título, resumo ou assunto, resultando na seleção de 21 estudos, dada sua relação com a saúde mental. A aplicação desta teoria se deu na abordagem profissional e usuário, assistência terapêutica, formação profissional, maternidade, paternidade, adoção e percepção pessoal de fenômenos como crise, vulnerabilidade social, doença, cuidado, self, exclusão social e violência. Compreender esses fenômenos possibilita a reflexão sobre a práxis de profissionais de saúde mental e afins, contribuindo com estratégias de acolhimento e formação de vínculo na relação entre profissional e usuário a partir da interpretação dos sentidos.

Palavras-chave: Assistência integral à saúde; Saúde holística; Psicologia social; Saúde mental; Comunicação; Linguagem. 


\begin{abstract}
This study has analyzed the application of discursive practices and production of meanings in the description and interpretation of the meanings given to different phenomena in mental health in Brazil. This is a bibliographical review based on specialized literature from the inquiry to selected scientific studies employing the Virtual Health Library (VHL) with the following keywords: 'discursive practices' and 'production of meanings', in the title, abstract or subject, resulting in the selection of 21 studies, given their relation to the mental health. The application of this theory is on the professional approach and user, therapeutic assistance, professional training, maternity, paternity, adoption and personal perception of phenomena such as crisis, social vulnerability, illness, care, self, social exclusion and violence. Understanding these phenomena enables the reflection about the praxis of mental health professionals and the like, contributing with strategies of integration and formation of links in the relationship between the professional and the user from the interpretation of the meanings.
\end{abstract}

Keywords: Comprehensive health care; Holistic health; Social psychology; Mental health; Communication; Language.

\title{
Resumen
}

Este estudio analizó la aplicación de las prácticas discursivas y producción de los sentidos en la descripción e interpretación de los sentidos dados a diferentes fenómenos en salud mental en Brasil. Se trata de una revisión bibliográfica basada en la literatura especializada, a partir de la consulta a estudios científicos utilizando la Biblioteca Virtual en Salud (BVS) con las palabras clave: 'prácticas discursivas' y 'producción de sentidos', en el título, resumen o asunto, resultando en la selección de 21 estudios, dada su relación con la salud mental. La aplicación de esta teoría se dio en el enfoque profesional y usuario, asistencia terapéutica, formación profesional, maternidad, paternidad, adopción, y percepción personal de fenómenos como crisis, vulnerabilidad social, enfermedad, cuidado, self, exclusión social y violencia. Comprender estos fenómenos posibilita la reflexión sobre la praxis de profesionales de salud mental y afines, contribuyendo con estrategias de acogida y formación de vinculo en la relación entre profesional y usuario a partir de los sentidos.

Palabras-clave: Atención integral de salud; Salud holística; Psicología social; Salud mental; Comunicación; Lenguaje.

INFORMAÇÕES DO ARTIGO

\begin{abstract}
Contribuição dos autores:
Concepção e desenho do estudo: Amanda Pereira Risso Saad, Geisa Alessandra Cavalcante de Souza e Paulo Roberto Haidamus de Oliveira Bastos;

Análise ou interpretação dos dados: Amanda Pereira Risso Saad e Geisa Alessandra Cavalcante de Souza;

Redação do manuscrito: Amanda Pereira Risso Saad, Geisa Alessandra Cavalcante de Souza e Paulo Roberto Haidamus de Oliveira Bastos; Revisão crítica do conteúdo intelectual: Amanda Pereira Risso Saad, Geisa Alessandra Cavalcante de Souza e Paulo Roberto Haidamus de Oliveira Bastos.
\end{abstract}

Declaração de conflito de interesses: não há.

Fontes de financiamento: concessão de bolsa da Coordenação de Aperfeiçoamento de Pessoal de Nível Superior (Capes) para a primeira autora.

Considerações éticas: não há.

Agradecimentos/Contribuições adicionais: não há.

Histórico do artigo: submetido: 10 set. 2018 | aceito: 20 maio 2019 | publicado: 12 set. 2019.

Apresentação anterior: não houve.

Licença CC BY-NC atribuição não comercial. Com essa licença é permitido acessar, baixar (download), copiar, imprimir, compartilhar, reutilizar e distribuir os artigos, desde que para uso não comercial e com a citação da fonte, conferindo os devidos créditos de autoria e menção à Reciis. Nesses casos, nenhuma permissão é necessária por parte dos autores ou dos editores. 


\section{Introdução}

A compreensão sobre o sentido dado a determinados fenômenos que participam da vida humana desperta interesse crescente para as ciências sociais contemporâneas. Analisar a aplicação das práticas discursivas e produção de sentidos, bem como apreender o(s) sentido(s) vinculado(s) ao cotidiano, mostram-se fundamentais para a compreensão e interpretação do processo dialógico inerente ao campo da saúde mental no cenário nacional.

As práticas discursivas estão diretamente relacionadas com o modo como as pessoas dão sentido às diferentes formas de interação presentes nas relações sociais do cotidiano, norteando a adoção de posicionamentos individuais. Seus principais elementos são a dinâmica (enunciados guiados por vozes), os speechgenres (discursos, linguagem social gerada pelos processos de institucionalização) e os conteúdos (repertórios interpretativos) ${ }^{1}$.

Como proposta teórico-metodológica do estudo da produção de sentido no cotidiano, esta abordagem vincula-se à psicologia social, utilizando a análise das práticas discursivas, focando a produção de sentido como construção dialógica e as diversas correntes que estudam a linguagem como práticas discursivas, à luz da perspectiva construcionista ${ }^{2}$.

A psicologia social, a partir dos estudos de Serge Moscovici sobre o senso comum, apresenta posicionamento no qual valoriza a observação e o estudo do comportamento humano em situações naturais do cotidiano em detrimento do estudo realizado em laboratórios. Ela pode ser definida como a ciência que privilegia a ligação do indivíduo com a sociedade, a partir de um espaço psicossocial em que é possível dialogar com elementos interdisciplinares aplicados ao entendimento de aspectos como a identidade, o discurso, a representação, a ação, entre outros ${ }^{3}$.

Na complexidade da interação humana, na qual os sentidos e os saberes são construídos e recriados constantemente a partir das práticas individuais e coletivas, encontra-se a mediação indivíduo-sociedade4

A base da psicologia social parte do pressuposto de que dar sentido ao mundo é algo poderoso e inerente à vida em sociedade. É um fenômeno sociolinguístico vinculado às práticas sociais que geram sentido, tais como as narrativas, argumentações e conversas. Possui relação íntima com os preceitos teóricos metodológicos que compõem as práticas discursivas e produção de sentidos, considerando a interpretação de repertórios individuais atrelados à linguagem social, valorizada em suas vertentes ${ }^{1}$.

Aliado à proposta teórico-metodológica das práticas discursivas e produção de sentidos, o construcionismo inaugurado pelos sociólogos Berger e Luckmann compreende que o sujeito e os objetos que compõem a realidade são considerados construções sócio-históricas, que devem ser constantemente analisadas a fim de possibilitar sua problematização e desfamiliarização. O conhecimento é percebido a partir da interação humana e das relações sociais, opondo-se à perspectiva hegemônica norte-americana da psicologia acadêmica que enfatiza os processos internos à mente, vinculados à cognição, visão também compartilhada pela psicologia social² ${ }^{2}$

De modo geral, as abordagens fundamentadas no construcionismo social apresentam posicionamento crítico do conhecimento sobre o que é a verdade e compreendem o mundo e os indivíduos como resultantes dos inter-relacionamentos pessoais, valorizando a história e a cultura. Tendem a descrever o mundo ou o self como produtos sociais, dando significado à linguagem de acordo com os diferentes modos de relacionamento, considerando-o como padrão de vida cultural5.

Neste contexto, as práticas discursivas e produção de sentidos mostram-se como potencial dispositivo aplicado ao estudo do(s) sentido(s) a partir do processo dialógico no âmbito da saúde mental, em especial, na relação entre profissional e usuário desses serviços. O aprimoramento das intervenções nesta área está intimamente ligado à Reforma Psiquiátrica, expressa pela instituição da Rede de Atenção Psicossocial no Brasil (RAPS), modelo de reorientação em saúde que contribui para a desinstitucionalização do sujeito, a partir da construção de territórios de cuidado que fomentem seu protagonismo ${ }^{6}$. 
Esta teoria pode ser utilizada como recurso leve e acessível, presente nas atividades em saúde, uma vez que se relaciona intimamente com o trabalho vivo decorrente do encontro profissional-usuário, cujos desdobramentos revelam singularidades dos momentos de fala, escuta e interpretações? .

Compreender a subjetividade, presente nos discursos produzidos nas relações humanas, mostra-se importante recurso para promover a ressignificação de elementos que participam do cuidado em saúde mental, bem como, possibilitar a reinserção do sujeito a partir do cuidado integral.

É salutar analisar as práticas discursivas e produção de sentidos na descrição e interpretação dos sentidos dados a diferentes fenômenos vinculados à saúde mental no cenário brasileiro em favor dos estudos do comportamento humano em situações espontâneas que considerem o saber do senso comum.

\section{Metodologia}

A metodologia utilizada foi a revisão bibliográfica baseada na literatura especializada por meio da consulta a estudos científicos selecionados a partir da Biblioteca Virtual em Saúde (BVS). A escolha de uma base de dados em saúde justifica-se devido ao objetivo deste estudo de analisar o uso das práticas discursivas e produção de sentidos no campo da saúde mental no Brasil. A aplicação desta teoria social para estudar os fenômenos em saúde favorece a articulação interdisciplinar e fomenta constante reflexão em direção às práticas profissionais inovadoras que considerem o ser humano em sua totalidade.

A análise das práticas discursivas e produção de sentidos no campo da saúde mental se deu a partir da categorização de unidades de exploração, a saber: objeto do estudo, metodologia e desfecho, cujo critério de busca correspondeu às palavras-chave 'práticas discursivas' e 'produção de sentidos' presentes no título, resumo e/ou assunto, consultados no período de agosto a novembro de 2017.

Utilizou-se o termo 'práticas discursivas' vinculado à produção de sentidos, cujo arcabouço teórico está ancorado na Psicologia Social e Construcionismo Social. Como critérios de inclusão, foram selecionados todos os artigos em língua portuguesa, disponíveis na íntegra para consulta, publicados de 2001 até 2017 e que utilizaram as práticas discursivas e produção de sentidos para descrever a percepção sobre fenômenos vinculados à saúde mental no Brasil. Foram excluídos todos os estudos que não estivessem relacionados com a saúde mental, a saber, aqueles vinculados ao estudo da percepção na área da comunicação e em ambientes organizacionais.

As bases de dados que estavam anexadas à BVS utilizadas foram: Lilacs (23), Index Psi - teses/Brasil (11); BDENF - enfermagem/Brasil (5); Index - Psi Periódicos técnico-científico (5); SES - SP Publicações Científico-técnicas (1); SES - SP Maternidade Leonor Mendes de Barros (1); SES - SP Instituto de Saúde (1) e Coleciona SUS/Brasil (1).

\section{Resultados}

$\mathrm{Na}$ busca avançada foram encontrados 48 resultados entre artigos, dissertações e teses em língua portuguesa que utilizavam as práticas discursivas e produção de sentidos como referencial teóricometodológico. No entanto, foram selecionados 11 artigos e 10 dissertações ou teses, por estarem relacionados à saúde mental.

A exploração dos estudos selecionados se deu a partir do encadeamento lógico entre o objeto do estudo, a metodologia e o desfecho pontuados por seus autores, no que tange à aplicação das práticas discursivas e produção de sentidos, como mostra a Figura 1: 


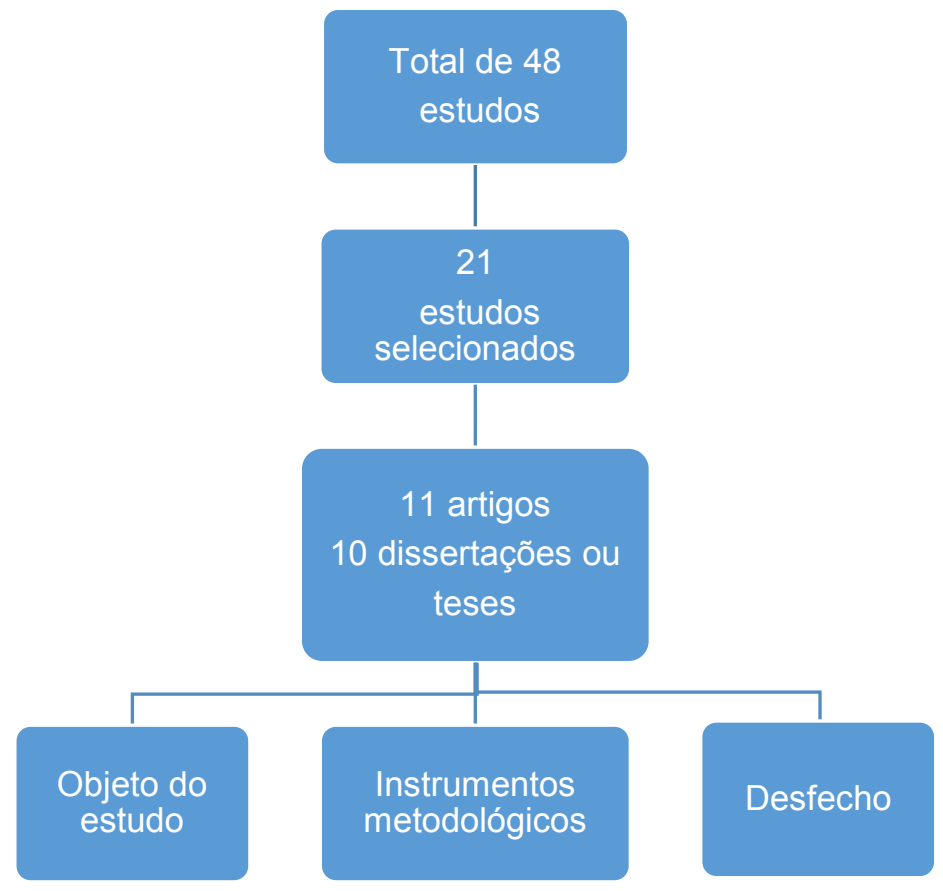

Figura 1 - Processo de escolha dos estudos analisados

Fonte: Os autores (2018).

No âmbito da saúde mental, esta teoria foi aplicada na abordagem entre profissional e usuário, assistência terapêutica, formação profissional, em fenômenos diversificados, a saber: maternidade, paternidade, adoção, crise, vulnerabilidade social, doença, cuidado, self, exclusão social e violência.

Para os estudos sobre a abordagem entre profissional e usuário, os instrumentos metodológicos utilizados foram: entrevista, audiogravação e estudo de caso. Entre os desfechos, tais estudos apontaram para a necessidade de novos padrões profissionais, com vistas à ressignificação de suas práticas, principalmente em relação à importância do acolhimento na conduta profissional ${ }^{8-12}$.

Para contemplar a assistência terapêutica aplicada à enfermagem obstétrica, foram utilizadas entrevista semiestruturada, análise documental e revisão bibliográfica. As considerações sobre esses serviços assistenciais também sugerem a necessidade de transformações das práticas cotidianas, principalmente em relação à desmedicalização e reestruturação dos serviços de saúde ${ }^{13,14}$.

A formação profissional de enfermeiros e psicólogos foi investigada utilizando entrevista semiestruturada, grupo focal e diário de campo. Observou-se que a avaliação desses profissionais necessita ser transformada a fim de romper com modelos que enfatizam a classificação diagnóstica e direcionar suas práticas profissionais para a formação multidisciplinar ${ }^{15-17}$.

Maternidade, paternidade e adoção representaram objetos de estudo no âmbito institucional e familiar, explorados a partir de entrevista clínica e/ou domiciliar e observação. O desfecho neste caso apontou para a identificação de formas singulares frente à maternidade, demanda por acompanhamento profissional pós-adoção, diversidade de sentidos atribuídos à maternidade por mulheres portadoras do Vírus da Imunodeficiência Humana (HIV-Human Immunodeficiency Virus) e necessidade de ressignificação do papel dos pais na relação com as filhas com transtornos alimentares ${ }^{18-21}$.

A percepção de fenômenos como crise e vulnerabilidade social emergiu de estudos relacionados às práticas profissionais em Centros de Atenção Psicossocial (CAPs) e Programa Hospital-Dia na perspectiva de seus usuários. Observou-se que a compreensão dos sentidos de periculosidade e cidadania influencia as práticas de atenção à crise, considerando que a representação destes profissionais sobre os usuários de programas de saúde mental estava vinculada à loucura e à prática medicamentosa. 
Há também fragilidades relacionadas à informação, bem como, inadequações na realização da testagem do HIV e divulgação dos resultados ${ }^{22-24}$.

Outrossim, fenômenos acerca de doença, cuidado, self, exclusão social e violência foram estudados a partir de entrevistas e encontros domiciliares, permitindo caracterizar a percepção sobre a 'doença dos nervos' como polissêmica e multifacetada. Já os sentidos sobre Doenças Sexualmente Transmissíveis (DST) e Síndrome da Imunodeficiência Adquirida (AIDS - Acquired Immunodeficiency Syndrome) estiveram vinculados à violência. Desse modo, pode se relacionar tais desdobramentos ao distanciamento entre reforma psiquiátrica e políticas para promoção de igualdades de raças e gênero, além de influência negativa do diagnóstico psiquiátrico na identidade, repercutindo em exclusão social nessa conjuntura ${ }^{25-28}$.

\section{Discussão}

\section{Comunicação profissional-usuário: ressignificando a interlocução}

A compreensão de sentidos em diversos contextos sociais a partir das práticas discursivas e produção de sentidos é aplicável na área educacional, bem como organizacional, hospitalar e na comunidade em geral.

No entanto, as publicações acerca desta teoria estiveram vinculadas, principalmente, às áreas de psicologia e enfermagem, nos contextos de saúde pública, patologia da fala/linguagem e toxicologia.

No campo da saúde mental, sua aplicação se deu em hospitais e Centros de Atenção Psicossocial (CAPs), cujas práticas profissionais, somadas na perspectiva da Estratégia de Saúde da Família, favoreceram importantes avanços frente à desinstitucionalização da pessoa acometida por doença mental no Brasil ${ }^{29}$.

Houve estudos que buscavam a compreensão de situações que envolviam a relação profissional e usuário, evidenciada, principalmente, nos trabalhos de enfermagem. Nesses estudos, os principais desfechos apontaram para a necessidade de adotar novas estratégias de coordenação na relação entre profissional/ usuário, de modo a contribuir com a proximidade e o bem-estar do paciente a partir da conduta do profissional de enfermagem, fundamentais ao acolhimento e formação de vínculo ${ }^{8-12}$.

Considerando a produção de sentido como fenômeno sociolinguístico, a busca pela compreensão das práticas discursivas e dos repertórios utilizados mostra-se salutar neste contexto ${ }^{1}$. Muito embora estes elementos sejam importantes na conduta do profissional, faz-se necessária uma visão holística do usuário, contemplando posturas éticas, sensíveis à realidade apresentada e que considerem o cenário clínico e epidemiológico na avaliação de riscos e estabelecimento de prioridades ${ }^{30}$.

Outrossim, o delineamento dos serviços de saúde deve contemplar estratégias que permitam identificar riscos potenciais aos seus usuários, a fim de que o gerenciamento das informações seja mediado pela interlocução entre a linguagem científica e a percepção sobre as diversas práticas associadas a tais riscos, e sentidos decorrentes da comunicação em saúde ${ }^{31}$.

A abordagem das práticas discursivas e produção de sentidos em estudo com casais soro discordantes, revelou sentimento de tristeza e revolta, fundamentados ora por suas experiências vividas, ora pela falta de acolhimento por parte dos profissionais de saúde ${ }^{31}$.

Sendo também uma construção sócio-histórica, os desdobramentos negativos, intimamente ligados à infecção pelo HIV, poderiam ser ressignificados, à medida que os profissionais aprimorassem suas competências emocionais voltadas ao acolhimento e à solidariedade em relação ao usuário, o que, de certa forma, representaria suporte para momentos de incerteza e solidão experenciados a partir do diagnóstico. Para isso, de acordo com Spink e Medrado ${ }^{1}$, seria necessário perceber a linguagem utilizada no trabalho como prática social e, desse modo, estabelecer novas versões de critérios de abordagem, que sejam compatíveis com um discurso humanizado por parte de seus interlocutores. 
A condução das abordagens profissionais ligadas à saúde mental deve contemplar a percepção da subjetividade do indivíduo. Esta dimensão, norteada pelos amplos princípios do Sistema Único de Saúde (SUS) e por outras políticas públicas apoiadoras, requer a reorientação das práxis frente à Reforma Psiquiátrica ${ }^{29}$.

A reestruturação da rede de atenção em saúde mental, ligada aos elementos constitutivos da atenção básica, fomenta o fortalecimento de aspectos como o desenvolvimento local e a territorialização, repercutindo na possibilidade de reinserção social e superação das limitações intimamente ligadas à doença mental ${ }^{29}$.

Outrossim, pesquisas evidenciaram essas mudanças nos serviços de saúde mental, refletindo em melhorias no modelo de saúde do Brasil, mais especificamente no contexto da assistência terapêutica, no qual a desmedicalização representa importante aspecto na transformação das práticas em saúde mental ${ }^{13-14}$.

Em favor das práticas avaliativas, tanto na área da enfermagem quanto da psicologia, alguns estudos destacaram a importância de uma formação que favoreça trabalhos multiprofissionais, bem como a necessidade de mudanças frente à atuação profissional, arraigada, ainda, ao modelo hegemônico de saúde, voltado à classificação das doenças e não ao indivíduo ${ }^{15,32}$.

Sobre este modelo, há demonstrações de que se mostra defasado e pouco efetivo no que tange à atenção à saúde de qualidade e humanização do cuidado. Além disso, destaca-se a importância da educação permanente, tendo por base o uso de tecnologias do campo da psicologia ${ }^{32}$.

Considerando a política nacional de saúde mental, o trabalho realizado nos CAPs deve transcender a multidisciplinaridade, impactando o plano terapêutico a partir de abordagens interdisciplinares ${ }^{33}$. Tais abordagens devem romper com o habitual para dar sentido aos discursos e favorecer ressignificações na interlocução².

\section{Percepção de fenômenos advindos do campo da saúde mental}

Diferentes aspectos psicossociais permearam o estudo da percepção de usuários de serviços de saúde em relação à maternidade, paternidade, adoção, crise, vulnerabilidade social, doença, cuidado, self, exclusão social e violência.

Modos de singularização e diversidade de sentidos foram atribuídos à maternidade por mulheres portadoras de HIV; modos esses vistos com um processo complexo que envolve percepções opostas, vinculadas à alegria e à tristeza, favorecendo reações inusitadas que demandam estratégias de acolhimento ${ }^{18-20,34}$. Considerando os elementos constitutivos das práticas discursivas, a dialogicidade, presente na abordagem dessas mulheres, pode construir sentidos positivos, fundamentais ao enfrentamento das demandas decorrentes da maternidade e da infecção pelo HIV, uma vez que, de acordo com Spink e Medrado², no diálogo habitam falantes e ouvintes, cujas percepções expressas em cada ato de fala são preditores de ressignificação.

Além do acolhimento, a continuidade do cuidado representada pelo acompanhamento por parte dos profissionais de saúde mental também contribui para ampliar os repertórios individuais que participam da relação mãe-filho nos casos de adoção, melhorando a estruturação dos vínculos ${ }^{21}$. Frente à relação paterna, identificou-se a necessidade de ressignificação do papel dos pais na relação com filhas portadoras de transtornos alimentares. A importância de se estabelecer ligações afetivas na relação entre pais e filhos também foi abordada, relacionando o vínculo familiar com o desenvolvimento de uma personalidade saudável ${ }^{35}$.

Em saúde mental, o conceito de crise merece atenção especial, principalmente se consideramos o sofrimento psíquico que envolve este processo. As práticas de atenção à crise devem ser repensadas em direção à cidadania do indivíduo que sofre e à diminuição de periculosidade que envolve a temática ${ }^{23}$. A atuação do profissional em saúde mental deve estar pautada na ética e no cuidado com a pessoa em crise, de modo a buscar a interpretação das práticas discursivas sob a perspectiva da atenção ao sofrimento psíquico e não apenas aos sintomas ${ }^{2,36}$. 
As representações dos profissionais vinculadas à loucura e à prática medicamentosa foram estudadas a partir de entrevistas domiciliares com os usuários de um Programa Hospital - Dia ${ }^{24}$. Os transtornos mentais geralmente estão acompanhados do uso de medicação, conforme discutido no estudo de Ferrazza ${ }^{37}$, o qual verificou que 99\% dos usuários de um programa de saúde mental eram medicados.

A loucura também esteve relacionada com a 'doença dos nervos', conceito comum presente nos serviços de saúde pública, atribuído, principalmente, a mulheres em condições socioculturais empobrecidas, conforme demonstrado por $\mathrm{Castro}^{25}$, que utilizou alguns encontros domiciliares para analisar os repertórios interpretativos desta população.

No ambiente de saúde mental o cuidado torna-se relevante nas relações entre profissional e usuário, e, nesse sentido, o estudo de Pedrosa ${ }^{26}$ pesquisou os significados de cuidado vinculados aos CAPs, principalmente em relação às questões de gênero. Ao analisar os textos técnicos do Ministério da Saúde, verificou-se nos discursos um distanciamento entre Reforma Psiquiátrica e programas de igualdade racial e de gênero, nos quais o sentido de cuidado está relacionado com o contexto terapêutico, em que a pessoa é vista em uma condição problemática ou de transtorno mental. O bem cuidar em saúde mental é consequência de inúmeros fatores associados, tais como a integração entre os programas de saúde mental, profissionais, usuários e seus familiares, a qual deve considerar o ambiente cultural, social e econômico dos envolvidos ${ }^{38}$.

Aragaki $^{27}$, ao analisar os discursos de usuários de serviços de saúde mental, buscou os sentidos de self e de pessoa e suas possíveis alterações após o diagnóstico psiquiátrico. Observou-se que este diagnóstico traz consigo a diminuição de trocas sociais, sendo marcado pela exclusão e submissão nos diferentes contextos sociais.

Outro aspecto referente a exclusão social foi abordado por Camarnado Junior ${ }^{28}$ ao expor o fenômeno da violência a partir de diversas análises macrossociais e estruturais. Utilizando entrevistas com usuários de programas e serviços de saúde com doenças sexualmente transmissíveis (DST), foi possível compreender os repertórios interpretativos sobre a violência.

Observou-se que os discursos estavam vinculados com preconceito e discriminação, expressos por meio de ações ou comportamentos velados e insidiosos. Nesse sentido, a violência repercute nas relações familiares e interpessoais, dificultando o diálogo e a boa convivência, impondo à vítima um papel de submissão, contrário à adoção de práticas sexuais seguras em relação às DST ${ }^{39}$.

Muito embora os serviços de saúde se mostrem permeados por densidades que participam da promoção e proteção da saúde, há de se considerar que tais serviços representam espaços privilegiados para a construção de saberes e exercícios das humanidades imbricadas nas relações. Desse modo, passa-se a perceber novas perspectivas em relação à vista, outrora explorada nessas densidades, o que permite emergir interlocutores mais habilitados para renovar os múltiplos jeitos de fazer saúde

\section{Conclusões}

As práticas discursivas e produção de sentidos em saúde mental no Brasil são utilizadas, principalmente, nas áreas de psicologia e enfermagem, cujas demandas estão diretamente relacionadas à valorização da subjetividade e à ruptura do modelo biomédico nas estratégias de comunicação em saúde.

Neste sentido, a valorização do diálogo repercute de maneira positiva frente à assistência terapêutica e favorece a possibilidade de compreensão de especificidades do usuário, com vistas à singularidade com que cada indivíduo responde ao sofrimento e tratamento.

Considerando que as relações sociais permitem a aquisição sistemática de experiências passíveis de reprodução e transformação, observa-se que esta teoria pode ser aplicada em diversos ambientes sociais e por diferentes áreas profissionais para apreender os sentidos produzidos por comunidades em geral, escolas, hospitais, clínicas e instituições públicas e privadas. Ela contempla aspectos psicossociais, a partir de práticas interdisciplinares, assegurando a construção de um cuidado singular, intransferível e inclusivo. 
A análise da aplicação desta teoria em psicologia social possibilita a reflexão sobre a práxis de profissionais de saúde mental, principalmente em direção às estratégias combinadas de acolhimento e formação de vínculo na relação entre profissional e usuário, de modo a permitir a apreensão de repertórios discursivos e sua reinterpretação, tendo em vista a necessidade de construir um cuidado holístico.

É mister a realização de estudos comprometidos com a identificação e análise do uso desta teoria em diferentes áreas do conhecimento, para possibilitar seu aprimoramento e novas aplicações no âmbito da interpretação dos processos dialógicos, presentes nos discursos que participam das relações sociais.

\section{Referências}

1. Spink MJ, Medrado B. Produção de sentidos no cotidiano: uma abordagem teórico-metodológica para análise das práticas discursivas. In: Spink MJ. Práticas discursivas e produção de sentidos no cotidiano: aproximações teóricas e metodológicas. São Paulo: Editora Cortez; 2013. p. 22-41.

2. Spink MJ, Frezza, MR. Práticas discursivas e produção de sentidos: a perspectiva da psicologia social. In: Spink MJ. Práticas discursivas e produção de sentidos no cotidiano: aproximações teóricas e metodológicas. São Paulo: Editora Cortez; 2013. p. 1-21.

3. Jovchelovitch S. Psicologia social, saber, comunidade e cultura. Rev Psicol Soc [Internet].2004 [citado em 2019 jul. 05];16(2):20-31. Disponível em: http://www.scielo.br/pdf/psoc/v16n2/a04v16n2

4. Traverso- Yépez M. Os discursos e a dimensão simbólica: uma forma de abordagem à psicologia social. Estud Psicol [Internet].1999 [citado em 2019 jul. 05];4(1):39-59. Disponível em: http://www.scielo.br/ pdf/epsic/v4n1/a04v04n1.pdf

5. Gergen KJ. Realities and relationships: soundings in social construction. Cambridge: Harvard University Press; 1994.

6. Ministério da Saúde (BR). Saúde mental em dados: informativo eletrônico de dados sobre a Política Nacional de Saúde Mental [Internet]. Brasília: Ministério da Saúde; 2015 [citado em 2019 jul. 05]. Disponível em: https://bit.ly/2ns3FqI

7. Merhy EE. $O$ ato de cuidar: a alma dos serviços de saúde [Internet]. In: Ministério da Saúde (BR). VERSUS Brasil: cadernos de textos. Brasília: Ministério da Saúde; 2004 [citado em 2019 jul. 05]. p.108-137. (Série B. Textos Básicos de Saúde). Disponível em: https://bit.ly/2FUBHiO

8. Aranda DAL. As dimensões do cuidado à criança: um diálogo entre famílias equipe de saúde na comunidade [tese na Internet]. Rio de Janeiro: Universidade Federal do Rio de Janeiro; 2003 [citado em 2019 jul. 05]. Disponível em: http://objdig.ufrj.br/51/teses/DeliaAuroraLazaroAranda.pdf

9. Costa AAS. Práticas discursivas na consulta de enfermagem [dissertação]. Fortaleza: Universidade Federal do Ceará; 2004.

10. Souza LV. Construindo cuidado a relação com os profissionais da saúde nas práticas discursivas de pessoas diagnosticadas com transtornos alimentares [tese na Internet]. São Paulo: Universidade de São Paulo; 2011 [citado em 2019 jul. 05]. Disponível em: https://bit.ly/2L5yxwH

11. Souza LV, Santos MA. Proximidade afetiva no relacionamento profissional-paciente no tratamento dos transtornos alimentares. Psicol Estud [Internet]. 2013 [citado em 2019 jul. 05];18(3):395-404. Disponível em: https://bit.ly/2Jf3fBw

12. Souza LV, Santos MA. Quem é o especialista? Lugares ocupados por profissionais e usuários no tratamento dos transtornos. Estud Psicol [Internet].2013 [citado em 2019 jul. 05];18(2):259-67. Disponível em: http://www.scielo.br/pdf/pe/v18n3/v18n3a01.pdf

13. Freitas HI. Estudos sobre diretrizes e práticas de atenção à saúde mental [dissertação na Internet]. São Paulo: Universidade Estadual Paulista Júlio de Mesquita Filho; 2006 [citado em 2019 jul. 05]. Disponível em: http://livros01.livrosgratis.com.br/cp025472.pdf

14. Gomes ML, Moura MAV, Souza IEO. A prática obstétrica da enfermeira no parto institucionalizado: uma possibilidade de conhecimento emancipatório. Texto Contexto-Enferm [Internet]. 2013 [citado em 2019 jul. 05];22(3):763-71. Disponível em: http://www.scielo.br/scielo.php?script=sci arttext\&pid $=$ S0104-07072013000300024

15. Ferraz CA, Laluna MCMC. Finalidades e função da avaliação na formação de enfermeiros. Rev Bras Enferm [Internet]. 2007 [citado em 2019 jul. 05];60(6):641-45. Disponível em: https://bit.ly/2Je9tl6 
16. Ferraz CA, Laluna MCMC. Os sentidos da prática avaliativa na formação de enfermeiros. Rev Latino-Am Enfermagem [Internet]. 2009 [citado em 2019 jul. 05];17(1):21-7. Disponível em: http://www.scielo.br/ pdf/rlae/v17n1/pt 04.pdf

17. Lima M, Santos L. Formação de psicólogos em residência multiprofissional: transdisciplinaridade, núcleo profissional e saúde mental. Psicol Ciênc Prof [Internet]. 2012 [citado em 2019 jul. 05];32(1):126-41. Disponível em: https://bit.ly/2FSOhiE

18. Castro CM. Os sentidos da maternidade: para gestantes e puérperas vivendo com HIV. [dissertação]. São Paulo: Pontifícia Universidade Católica de São Paulo; 2001.

19. Moura SMSR, Araújo MF. Produção de sentidos sobre a maternidade: uma experiência no programa mãe canguru. Psicol estud [Internet]. 2005 [citado em 2019 jul. 05];10(1):37-46. Disponível em: https://bit. Iy/2NBYjuH

20. Saviolli CMG. Construção de sentidos sobre a participação do pai no tratamento de filhas com diagnóstico de transtornos alimentares [dissertação na Internet]. São Paulo: Universidade de São Paulo; 2012 [citado em 2019 jul. 05]. Disponível em: https://bit.ly/2xuZeCl

21. Costa NRA, Rossetti-Ferreira MC. Tornar-se pai e mãe em um processo de adoção tardia. Psicol Reflex Crít [Internet] .2007 [citado em 2019 jul. 05];20(3):425-34. Disponível em: https://bit.ly/30gaH5a

22. Fernandes NM, Hennington EA, Bernardes JS, Grinszdejn BG. Vulnerabilidade à infecção de HIV entre casais sorodiscordantes no Rio de Janeiro, Brasil. Cad Saúde Pública [Internet]. 2017 [citado em 2019 jul. 05];33(4):1-12. Disponível em: http://www.scielo.br/pdf/csp/v33n4/1678-4464-csp-3304-e00053415.pdf

23. Willrich JQ, Kantorski LP, Chiavagatti FG, Cortes JM, Pinheiro GW. Periculosidade versus cidadania: os sentidos da atenção à crise nas práticas discursivas dos profissionais de um Centro de Atenção Psicossocial. Physis [Internet]. 2011 [citado em 2019 jul. 05];21(1):47-64. Disponível em: http://www. scielo.br/scielo.php?script=sci arttext\&pid=S0103-73312011000100004

24. Guareschi NMF, Reis C, Oliven T, Hüning SM. Usuários do Hospital-Dia: uma discussão sobre performatividade em saúde e doença mental. Rev. Mal-Estar Subj [Internet]. 2008 [citado em 2019 jul. 05];8(1):119-37. Disponível em: http://www.scielo.br/scielo.php?script=sci arttext\&pid $=\mathrm{S} 0104-07072013000300024$

25. Castro CA. Dando conta da "doença dos nervos": produção de sentidos em conversas com mulheres [dissertação na Internet]. Fortaleza: Universidade Federal do Ceará; 2010 [citado em 2019 jul. 05]. Disponível em: http://www.repositoriobib.ufc.br/000032/00003293.PDF

26. Pedrosa CH. Cuidar? Sim; olhar de gênero? Não. Os sentidos do cuidado no CAPS em documentos técnicos do Ministério da Saúde [dissertação]. São Paulo: Pontifícia Universidade Católica de São Paulo; 2006.

27. Aragaki SS. O aprisionamento de selves em diagnósticos na área de saúde mental. [dissertação na Internet]. São Paulo: Pontifícia Universidade Católica de São Paulo; 2006 [citado em 2019 jul. 05]. Disponível em: https://bit.ly/2XSOzBS

28. Camarnado Junior DV. Os sentidos da violência nos programas e serviços de saúde em DST/AIDS [tese na Internet]. São Paulo: Programa de Pós-Graduação em Ciências da Coordenadoria de Controle de Doenças da Secretaria de Estado da Saúde de São Paulo; 2005 [citado em 2019 jul. 05]. Disponível em: ses.sp.bvs.br/lildbi-textos/docsonline/get.php?id=442

29. Ministério da Saúde (BR). Saúde mental e atenção básica: o vínculo e o diálogo necessários [Internet]. Brasília: Ministério da Saúde; 2003 [citado em 2019 jul. 05]. Disponível em: https://www.nescon. medicina.ufmg.br/biblioteca/imagem/1734.pdf

30. Tesser CD, Poli Neto P, Campos GWS. Acolhimento e (des)medicalização social: um desafio para as equipes de saúde da família. Ci Saúde Coletiva [Internet]. 2010 [citado em 2019 jul. 05];15(3):3615-24. Disponível em: $\underline{\text { http://www.scielo.br/scielo.php?script=sci arttext\&pid=S1413-812320100000900036 }}$

31. Spink MJ. Contribuições da psicologia discursiva para o campo da comunicação sobre riscos em saúde. R. Eletron. Comunic. Inf. Inov. Saúde [Internet]. 2019 jan-mar [citado em 2019 jul. 05];13(1). Disponível em: https://www.reciis.icict.fiocruz.br/index.php/reciis/article/view/1749/2248

32. Mendes EV. As redes de atenção à saúde. 2. ed. Brasília: Organização Pan Americana de Saúde; 2011 [citado em 2019 jul. 05]. Disponível em: https://bit.ly/2L2SAMo 
33. Schneider ARS. A rede de atenção em saúde mental: a importância da interação entre atenção primária e os serviços de saúde mental. Ci Saúde [Internet]. 2009 [citado em 2019 jul. 05];2(2):78-84. Disponível em: https://bit.ly/2XOzzDI

34. Carvalho FTC, Piccinini CA. Aspectos históricos do feminino e do maternal e a infecção pelo HIV em mulheres. Ci Saúde Coletiva [Internet]. 2008 [citado em 2019 jul. 05];13(6):1889-98. Disponível em: https://bit.ly/2LGY3bd

35. Wellausen RS, Bandeira DR. O tipo de vínculo entre pais e filhos está associado ao desenvolvimento de comportamento antissocial? Interam J Psychol. 2010;44(3):498-506.

36. Jardim K, Dimenstein M. Risco e crise: pensando os pilares da urgência psiquiátrica. Psicol Ver [Internet] 2007 [citado em 2019 jul. 05];13(1):169-90. Disponível em: https://bit.ly/2RWczwV

37. Ferrazza $D A$, Luzio $C R$, Sanches RR. A banalização da prescrição de psicofármacos em um ambulatório de saúde mental. Paidéia [Internet]. 2010 [citado em 2019 jul. 05];20(47):381-90. Disponível em: https://bit.ly/2NykPol

38. Cardoso L, Galera SAA. O cuidado em saúde mental na atualidade. R Esc Enferm USP [Internet]. 2011 [citado em 2019 jul. 05];45(3):687-91. Disponível em: https://bit.ly/2RXIWLK

39. Ruzany MH, Taquette SR, Oliveira RG, Meirelles ZV, Ricardo IB. A violência nas relações afetivas dificulta a prevenção de DST/AIDS? J Pediatr [Internet]. 2003 [citado em 2019 jul. 05];79(4):349-54. Disponível em: http://www.scielo.br/pdf/jped/v79n4/v79n4a14.pdf 